\title{
ÜBERSICHT
}

\section{Professionelle polizeiliche Kommunikation: sich verstehen}

\author{
Mario S. Staller ${ }^{1}$ (D) Swen Koerner ${ }^{2}$ D $\cdot$ Benjamin Zaiser ${ }^{3}$
}

Eingegangen: 29. Juli 2021 / Angenommen: 17. September 2021 / Online publiziert: 7. Oktober 2021

C C Der/die Autor(en) 2021

\section{Zusammenfassung}

Auch wenn, einem Bonmot Watzlawicks folgend, nicht nicht kommuniziert werden kann, kann Kommunikation gleichwohl mehr oder weniger viel Aufmerksamkeit zuteilwerden. In den modernen Sozialwissenschaften ist Kommunikation längst theoretisch und empirisch zum Schlüsselelement komplexitätsangemessener Beschreibungen und Erklärungen moderner Gesellschaftsvollzüge avanciert. In der modernen Polizeiarbeit hat der aktuelle Wissensbestand zur Kommunikation diesen Anspruch noch nicht erreicht. Der Beitrag setzt hier an, indem er polizeiliche Kommunikation als zentrales Medium von Polizei-Bürger*innen-Interaktionen pragmatistisch modelliert. Demensprechend hat die Polizei in ihrer Kommunikation dem der Öffentlichkeit geschuldeten Maß an Professionalität gerecht zu werden. Diese, so unser zentrales Argument, zeigt sich als Kontrolle durch Einsicht in potenziell „blinde Flecken“ polizeilicher Kommunikation.

Schlüsselwörter Systemische Kommunikation · Professionalität · Reflexivität · Gewalthandeln ·

Polizei-Bürger*innen-Interaktion

\section{Reflective communication for a professional police}

\begin{abstract}
According to Watzlawick (2017), one cannot not communicate (p. 59). However, when humans interact, they are not always aware of the way they communicate with each other. Interpersonal communication has a broad theoretical and empirical foundation in modern social scientific literature, which reflects its complexity as a concept at the center of our understanding of social practice. In police sciences and the study of law enforcement, communication has not yet been examined and acknowledged at that level. To address this shortfall, this contribution presents a pragmatic argument to understand interpersonal communication at the center of all police-citizen encounters. As a consequence, the public can expect police to be aware of the crucial impact their communication has and perform at a level of corresponding professionalism.
\end{abstract}

Keywords Systemic communication $\cdot$ Professionality $\cdot$ Reflexivity $\cdot$ Violent action $\cdot$ Police-Citizen-Interaction

Prof. Dr. mult. Mario S. Staller

mario.staller@hspv.nrw.de

1 Fachbereich Polizei, Hochschule für Polizei und öffentliche Verwaltung Nordrhein-Westfalen, Aachen, Deutschland

2 Abteilung für Trainingspädagogik und Martial Research, Deutsche Sporthochschule Köln, Köln, Deutschland

3 Department of Psychological Sciences, University of Liverpool, Liverpool, Großbritannien

\section{Einleitung}

Kommunikation ist eine Herausforderung. Insbesondere Angehörige von Berufen, in denen das Bewältigen von Konflikten Teil der Profession ist, sehen sich Kommunizierende der Herausforderung gegenüber, sich als Anwender*in die entscheidenden Theorien und Modelle anzueignen. Dies wiederum setzt Aus- und Fortbildungssowie Trainingsstrukturen innerhalb der beruflichen Praxen unter Druck: Welches Modell soll vermittelt werden? Sind die 4 Von-Thun'schen-Ohren (von Thun 1981) wichtiger als das Treppenstufenmodell von Vecchi (Vecchi et al. 2005, 
2019) oder die Kommunikationsaxiome von Watzlawick (Watzlawick et al. 1967)? Wie steht es um die gewaltfreie Kommunikation (Rosenberg 2003), das aktive Zuhören (Rogers und Farson 1957), die Validation (Feil 1989) oder um die Transaktionsanalyse (Berne 1961)? Welche darunterliegenden Konzeptionen von Kommunikation sollen Anwendung finden? Liegt der Fokus auf verbaler Kommunikation oder finden Handlungen - wie Gewalthandeln bei der Polizei - auch Eingang in die Betrachtung (Koerner und Staller 2021a). Wird das darunterliegende Verständnis von Luhmanns systemtheoretischer Kommunikationstheorie (Luhmann 1981), dem kommunikativen Konstruktivismus (Reichertz 2010, 2020) oder sozialpsychologischen Sender-Empfänger-Modellen (für einen Überblick: Krauss und Fussell 1996) getragen? Und wie verhält es sich mit der Übertragung in die Handlungspraxis? Welche Handlungsempfehlungen werden gegeben? Was könnte wie in welcher Situation wie kommuniziert werden, um das beabsichtigte Ergebnis zu erreichen?

Die Anwendungspraxis weist auf mehrere Problematiken hin: Reibungs- und Transferverluste zwischen Inhalten der kommunikationstheoretischen Literatur und dem polizeilichen Einsatztraining (Hermanutz und Spöcker 2012) sowie zwischen Einsatztraining und Einsatzfall (Staller et al. 2021b). Interessanterweise erwähnten Polizist*innen, die danach gefragt wurden, was in Konfliktsituationen gut funktioniert und was nicht, keine Kommunikationstheorien oder -modelle (Staller et al. 2021b). Insofern stellt sich die Frage, ob und ggf. inwieweit Kommunikationstheorien und -modelle einen Unterschied in der polizeilichen Praxis machen. Wirkungsstudien dazu sind uns nicht bekannt. Dennoch weisen empirische Befunde weltweit - so auch in Deutschland - auf Problematiken in der sozialen Interaktion sowie in der Konfliktbearbeitung mit Bürger*innen hin (Boxer et al. 2021). Dies deutet auf eine Kluft zwischen modelltheoretischer Lehre, zumeist in psychologischen Lehrveranstaltungen von Polizeianwärter*innen, und dem Anwendungsfall in der Praxis bzw. in der Einsatzsituation hin (Staller und Koerner 2021d).

Die Ausdifferenzierung der Forschung mit spezifisch relevanten Fragestellungen sowie praktischen Modellen, Empfehlungen und Techniken, die Kommunikation zum Thema haben, lässt das Thema immer komplexer erscheinen - und nähert sich vermutlich damit der Realität des Gegenstandes an: Kommunikation als komplexes Phänomen. Der Markt der (Modell-, Theorie- und Technik-)Möglichkeiten scheint schier unbegrenzt. Das Treffen einer Auswahl für den professionellen Trainings- oder Schulungsprozess aber auch in der Anwendung - ist final. Abhängig von dem, was ausgewählt wird, werden andere Möglichkeiten ausgeschlossen und geraten damit - auch in der Anwendung aus dem Blick. Gleiches gilt auch für den Fokus in Bezug auf die vermittelte Kommunikationstheorie, das Kommu- nikationsmodell oder die Kommunikationstechnik per se: Die Betrachtung von dem, was stattfindet (Kommunikation), lässt die Produktion eben dieser aus dem Blick geraten. In dieser Hinsicht kommt der polizeilichen Aus- und Fortbildung damit eine wichtige Funktion zu. Sie soll über deklarative Wissensstrukturen Reflexivität ermöglichen und so zu einer professionellen Polizeiarbeit beitragen. Bei der Auswahl dieser Wissensbestände entsteht allerdings notwendigerweise ein Ausschlussbereich, der seinerseits selbst Reflexivität - und damit zuerst Beobachtung - erfordert. Die Auswahl bestimmter Wissensbestände schließt stets die Vermittlung anderer Wissensbestände aus. In Bezug auf kommunikatives Handeln stellt sich entsprechend die Frage: Was könnte über Kommunikation kommuniziert werden, das die Problemlösung in der beruflichen Praxis unterstützen könnte? Hierin besteht der Ausgangspunkt unserer Überlegungen.

Ausgehend von einer systemtheoretischen Modellierung polizeilicher Kommunikation, nehmen wir unter Augenmerk auf die in der Praxis prävalenten Probleme 6 blinde Flecken in den Blick. Diese können in allgemein formulierten Kommunikationstheorien und -modellen aufgrund ihres Abstands zum Forschungsgegenstandes (Polizieren) nicht in den Blick genommen werden. Die Bearbeitung dieser blinden Flecken qualifizieren wir als professionelle polizeiliche Kommunikation.

\section{Polizeiliche Kommunikation}

In Übereinstimmung mit der Theorie moderner sozialer Systeme (Luhmann 1990) und in struktureller Analogie zu pädagogischer Kommunikation in Lehr-Lern-Settings (Kade 2004; Körner und Staller 2018) identifizieren wir Kommunikation als elementares Ereignis polizeilicher Einsatzsituationen (Koerner und Staller 2021a; Luhmann 1990). Wir verzichten an dieser Stelle auf eine komplizierte Diskussion zur Beziehung der Systemtheorie zum Menschen als individuellen Akteur (für eine Zusammenfassung: Körner 2017). Stattdessen nutzen wir die Systemtheorie als heuristische Grundlage für eine pragmatistische Modellierung (Rorty 2003) polizeilicher Kommunikation. Kommunikation besteht dabei aus einer dreistelligen Selektion: der Auswahl einer Information (1) sowie einer Mitteilungsform aufseiten des alter (2) sowie einer Anschlussselektion aufseiten des ego (3) (die ihrerseits Mitteilung einer Information ist). Kommunikation phänomenalisiert sich letztlich über Mitteilung (Luhmann 1995), indem sie als verbale und körperliche Äußerung in Erscheinung tritt. In der polizeilichen Kommunikation kommen dabei verschiedene Dimensionen zum Tragen. 
1. Der Prozess der polizeilichen Kommunikation wird durch zwei Prämissen untermauert. Er geht (a) von einer Asymmetrie kommunikativer Voraussetzungen aus. Jemand (alter) verfügt über Durchsetzungskönnen mittels legitimierter Gewalt, während die andere Seite (ego) dies nicht kann. Weiterhin geht polizeiliche Kommunikation (b) von der Annahme eines Veränderungspotenzials aus: etwas am Verhalten des ego ist nicht so, wie es sein sollte, aber sein könnte. Die Umsetzung des individuellen Potenzials in die Realität, eine Verhaltensanpassung durch $e g o$, ist ein wesentliches Ziel polizeilicher Kommunikation.

2. In der sozialen Dimension konstituiert sich polizeiliche Kommunikation durch alter und ego, die als Interagierende wechselseitig aufeinander bezogen sind und je nach Perspektive entweder die Rolle von ego oder alter einnehmen.

3. In der Sachdimension geht es in der polizeilichen Kommunikation um Informationen, die eine Problemlösung ermöglichen sollen. An dieser Stelle erfolgt eine doppelte Selektion durch das alter: Welche Information soll mitgeteilt werden (dies/nicht das), und wie soll diese mitgeteilt werden (mündlich, schriftlich, gestisch, mit Gewalteinsatz, mit Lautsprecher, über Social Media etc.)?

4. In der zeitlichen Dimension konstituiert sich polizeiliche Kommunikation durch zwei aufeinander bezogene Operationen: Mitteilung von Information (durch alter) und Anschlusshandeln (z. B. Aneignung) durch ego.

5. Polizeiliche Kommunikation findet im gesamtgesellschaftlichen Kontext statt. Die Gesellschaft ist Voraus- setzung und Folge zugleich: Voraussetzung, da Umgangsweisen gesellschaftlich eingeübt und verbürgt sind, und Folge, da sich die Gesellschaft immer wieder aufs Neue durch Kommunikation gestaltet und reproduziert (Abb. 1).

Polizeiliche Kommunikation findet in unterschiedlichen zeitlichen und räumlichen Kontexten statt: in einer spezifischen Situation in einer Begegnung zwischen Bürger*in und Polizist*in, in polizeilichen Gruppenlagen sowie zwischen der Polizei als Institution und der Zivilgesellschaft als Ganzes.

Die Polizei sieht sich in der jüngsten Zeit einer besonders durch mobile Videoaufnahmen ermöglichten öffentlich diskutierten Kritik ausgesetzt (Schuck 2019), die international auch seitens der Wissenschaft erhöhter Aufmerksamkeit ausgesetzt ist (Bennell et al. 2021; Boxer et al. 2021). Im Ergebnis weisen Befunde auf eine teilweise deprofessionalisierte polizeiliche Kommunikation hin (für eine Übersicht: Boxer et al. 2021).

Deprofessionalisiert verstehen wir als Gegenpol zu einer ausgeübten Professionalität, die wir als berufsförmige, gesetzlich mandatierte und berufsethisch reflektierte Ausübung einer Tätigkeit von essenzieller gesellschaftlicher Bedeutung verstehen (Werner 2019). Befunde aus der polizeilichen Praxis weisen allerdings auf systematische Probleme in Bezug auf das Umsetzen einer reflexiven Sichtweise auf das eigene Handeln hin.

Eine professionelle polizeiliche Kommunikation ist sich der Tatsache bewusst, dass das Einnehmen einer bestimm-

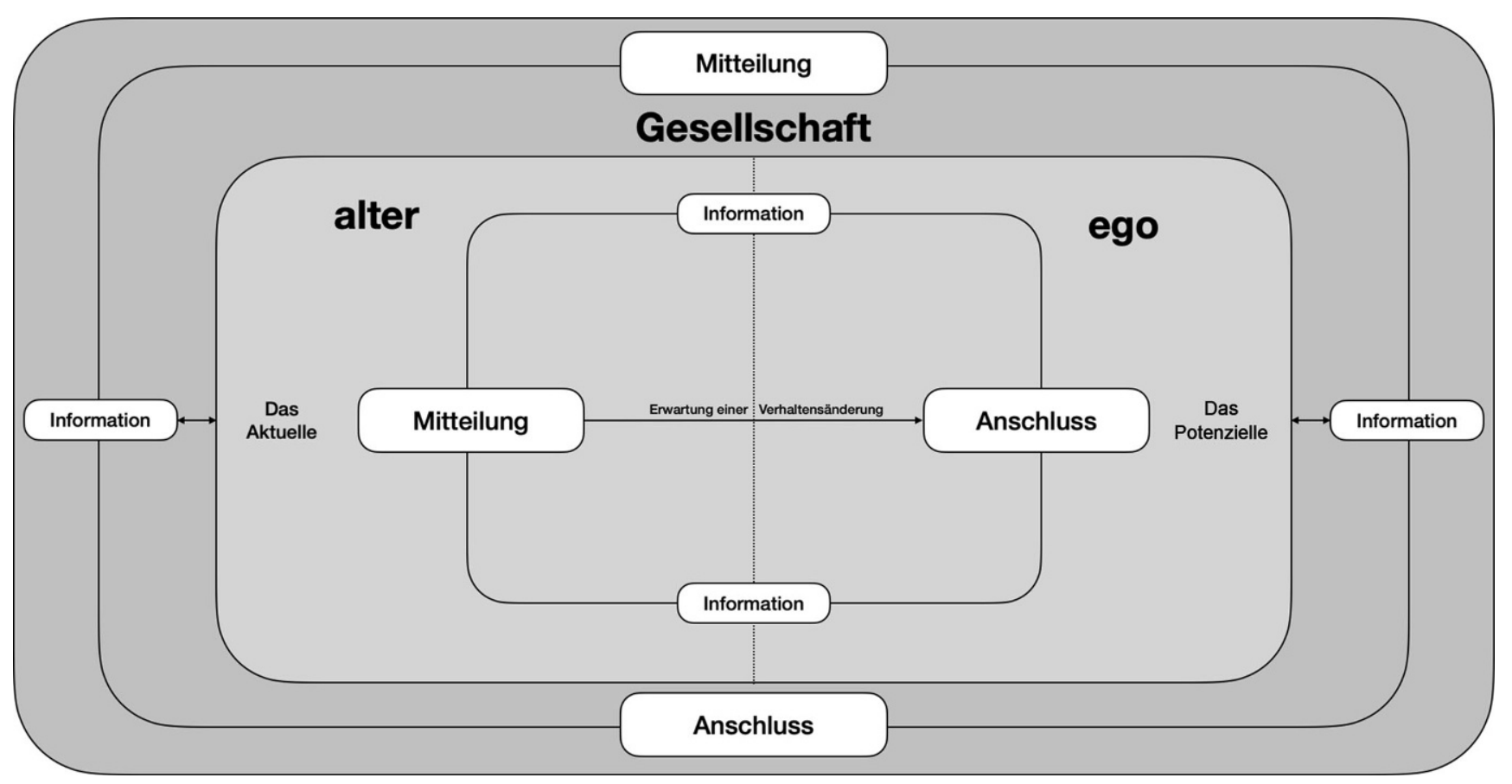

Abb. 1 Modell polizeilicher Kommunikation 
ten Perspektive notwendigerweise blinde Flecken mit sich bringt. Professionelle polizeiliche Kommunikation qualifiziert sich durch die Reflexion auf ebensolche, fallweise auftretenden Ausschlussbereiche, die das Sehen ermöglichen, aber im Moment des Sehens selbst nicht beobachtet werden können (Staller und Koerner 2021a). Aus praktischer Perspektive gilt es folglich, die aktuellen blinden Flecken aufzuzeigen und einer systematischen Bearbeitung zuzuführen. In Bezug auf professionelle polizeiliche Kommunikation identifizieren wir ohne Anspruch auf Vollständigkeit die nachfolgenden ,,blinden Flecken“.

\section{Blinder Fleck 1: Kommunikation und Entscheidungsverhalten}

Kommunikation ist funktional, insofern bestimmt werden kann, welches Problem sie löst. In beruflichen Kontexten, so auch in der Polizei, dient sie häufig dazu, Probleme zu lösen, wie z. B. konfliktgeladene Situationen zu deeskalieren, Maßnahmen zu erklären und durchzusetzen, im Rahmen von Großereignissen zu informieren oder Strategien mitzuteilen und zu begründen. Mit anderen Worten: Es existiert eine klare Problemstellung, die mithilfe von Kommunikation bearbeitet werden soll. Hier werden zwei Aspekte deutlich:

Erstens, die Funktionalität der Kommunikation in Bezug auf Handlungsprobleme (Reichertz 2020): „Kommunikation existiert nicht deshalb, damit Menschen ihr Innerstes einem Gegenüber möglichst genau übermitteln können, sondern Kommunikation dient der Lösung von Handlungsproblemen und der Handlungskoordinierung“ (S. 21). Allerdings stellt sich dieses „Bewirken“ nicht immer einfach dar. Die der Kommunikation innewohnenden Unsicherheiten hängen zum einen mit den unterschiedlichen Erwartungen und Wünschen von Kommunikationssteilnehmer*innen zusammen, zum anderen mit der prinzipiellen Möglichkeit des Nichtverstehens: (a) in Bezug auf das Nichtteilen von Geltungsansprüchen gewisser Normvorstellungen und (b) in Bezug auf die angewandten kommunikativen Akte (Reichertz 2010). Zweitens, dieses Bewirken, wenngleich mit Unsicherheiten behaftet, stellt eine Utilisierung der Kommunikation durch alter dar, um die oben besprochene Problemlösung zu erreichen. Damit wird ein Aspekt polizeilicher Kommunikation deutlich, der eine weitere Unsicherheit aufweist: Die Selektionen der Kommunikation (pragmatistisch - aber systemtheoretisch nicht korrekt formuliert: die kommunikativen Handlungen) sind Entscheidungsprozesse und damit dem menschlichen Urteils- und Entscheidungsverhalten unterworfen - und als solche kognitiven Verzerrungen und Fehlurteilen ausgesetzt.

Polizeiliches Verhalten - und damit Kommunikation (s. Abschn. „Blinder Fleck 3“) - in Interaktions- und Konfliktsituationen ist unterschiedlichen kognitiven Verzerrun- gen und Fehlurteilen unterworfen (ausführlich: Staller et al. 2021f). Das Problem hierbei scheint die Verwobenheit von Verzerrungen und kognitiven Fehlschlüssen auf individueller als auch organisationaler Ebene, welche sich Gegenseitig stabilisieren und eine reflexive Bearbeitung erschweren. Fehlschlüsse über die Natur der kognitiven Fehlschlüsse erschweren deren Erkennung und Bearbeitung, während diese gleichzeitig wieder Fehlschlüsse bestärken. Im Kern führt dies dazu, dass menschliches, in Organisationen eingebettetes Entscheidungsverhalten massiven Fehlurteilen unterliegt, denen es strukturell zu begegnen gilt. Kommunikation als auf Entscheidungen basierende Prozesse ist damit genauso diesen Fehlurteilen unterworfen.

Professionelle Polizeiliche Kommunikation ist sich den sie beeinflussenden kognitiven Verzerrungen und Fehlurteilen bewusst.

\section{Blinder Fleck 2: lineare Kausalitäten und kausaler Durchgriff}

Polizei-Bürger*innen-Interaktionen sind ein hochkomplexes Phänomen. Die stattfindende polizeiliche Kommunikation ist dabei geprägt von einer hochgradig komplexen Interaktion der beteiligten, aber füreinander auf der Ebene des Bewusstseins operativ unerreichbaren Systeme (alter, ego, Interaktion). Daraus folgt, dass die Mitteilung einer Information nicht automatisch zu Verständnis und Annahme führt und damit kein kausaler Durchgriff seitens alter auf ego möglich ist.

Die Problematik wurde für das schulische Unterrichten von Luhmann und Schorr (Luhmann und Schorr 1982) als „Technologiedefizit“ beschrieben. Es gibt eben in sozialen Interaktionen nicht die einfache Technologie wie in anderen Bereichen menschlichen Schaffens (Computertechnik, Mechanik, Pharmazie, etc.), die wie eine App, ein Knopf oder eine Pille planmäßig zum gewünschten Ergebnis führt. Für das polizeiliche Einsatzhandeln legen Analysen nahe, dass das inhärente Technologiedefzit über eine Kompensation durch finalisiertes Wissen bearbeitet wird: der kausale Durchgriff über „,kausale Mechanismen“ (Kern 2017, S. 56) wie sie beispielsweise in polizeilicher Kommunikationsliteratur vermittelt werden (Kern 2017): „Von der Senderin bzw. vom Sender wird die Wirkung auf die Empfängerin oder den Empfänger wahrgenommen. Es kommt demnach zu Rückkoppelungen, weil sich die sendende Person selbst je nachdem, ob es ihr gelungen ist, die gewollten kommunikativen Ziele durchzusetzen, beispielsweise als kompetent oder inkompetent erlebt“" (S. 56).

In Bezug auf das Konfliktlösungsverhalten wird im Rahmen der Konstruktion der ,aggressiven Polizeimännlichkeit" (Seidensticker 2021) die körperliche Überlegenheit von Männern zur Reduktion der Komplexität sozialer Konfliktprozesse herangezogen. Wer stärker ist, kann den Kon- 
flikt leichter lösen. Die Konstruktion derartiger kausaler Zusammenhänge trägt maßgeblich dazu bei, Konflikte leichter deut- und bearbeitbarer zu machen.

Im Kontrast dazu beschreibt eine komplexitätstheoretische Sichtweise (Luhmann 1984) Kommunikation als Selektionsprozesse auf verschiedenen Ebenen (Information, Mitteilung, Verstehen), welche im Einklang miteinander und simultan von alter und ego bezogen aufeinander durchgeführt werden. Welche Selektionen von wem nach welchen Relevanzkriterien durchgeführt werden, ist nicht absolut vorhersehbar.

Auch tradierte Einsatzmodelle legen einen linearen Kausalzusammenhang im polizeilichen Kommunikationsverhalten nahe (Bernt und Kuhleber 1991), der auch noch so in der polizeilichen Bildungspraxis gelehrt wird. Das neu entwickelte Gewaltreduzierende Einsatzmodell (GeredE; Staller et al. 2021e) nimmt diese komplexitätstheoretischen Überlegungen ernst und verwehrt sich einer linearkausalen Sichtweise. Der Umgang mit Komplexitätsbedingungen erfordert eine andere Logik: Anstelle eines kausalen Durchgriffs steht eine probabilistische Möglichkeit der Einflussnahme über die Gestaltung der Kommunikation. Die Orientierung an variablen Faktoren und Ereignissen sowie das Nutzen von situationsrelativen Kausalplänen steht anstelle vorgefertigter Handlungsmuster, die sich durch Abstrahierung individuellen Kontexten entziehen.

Der Wunsch nach linear-kausalen Denkmodellen ist nachvollziehbar: Sie vermitteln Kontrolle. Wenn A, dann B. Sofern also A, ist das Ergebnis (B) kontrollierbar. Das hat einen intuitiven Anziehungscharakter. Die Logik von komplexen Systemen ist weniger intuitiv. Es stellt sich ein wahrgenommener Kontrollverlust ein. Und gerade Studien in Bezug auf polizeiliche Stressoren weisen auf Unsicherheit und Kontrollverlust als Stressoren polizeilicher Praxis hin (Frenkel et al. 2020, 2021).

Komplexitätsorientierte Denkmodelle haben einen anderen Kontrollmechanismus: Einsicht (Nassehi und Saake 2002). Einsicht wie die Dinge zueinanderstehen, welche Faktoren und Variablen Einfluss auf bestimmte Effekte haben. Und Einsicht in die kontingente Natur der Dinge: dass es so ist, wie es ist - aber auch anders sein könnte (Körner 2009; Staller und Koerner 2021b). Dies erfordert entsprechend umfängliche Wissensstrukturen (Berkes und Berkes 2009) und ein reflektiertes Herangehen an Problemstellungen der täglichen Praxis (Schön 1983), um die Einsichten systematisch zu vergrößern. Für die polizeiliche Kommunikation legt das GeredE (Staller et al. 2021e) eine derartige Struktur dar: Anhand von 6 Wissensdimensionen (Wer, Was, Wie, Selbst, Kontext, Planungs- und Reflexionspraxis), die gleichsam als Reflexionspunkte für situative Entscheidungen genutzt werden können, weist das Gere$\mathrm{dE}$ Bereiche aus, die den Verlauf der Kommunikation (und darunter gefasst des Einsatzes) beeinflussen. Eine komple- xitätsorientierte Herangehensweise an polizeiliche Kommunikation ist sich dieser Abhängigkeiten bewusst, arbeitet an tieferen Einsichten innerhalb der Dimensionen und ihren Verbindungen und setzt die Einsichten situationsvariabel begründet ein. Mit dem GeredE liegt damit ein Alternativmodell zu dem bisher sich in Vorschriften wiederfindenden Deeskalierenden Einsatzmodell (Bernt und Kuhleber 1991; Leitfaden 371 2011) vor, welches tendenziell von einer linearen Kausallogik ausgeht (für eine ausführliche Kritik: Staller et al. 2021e).

Professionelle Polizeiliche Kommunikation bedient sich komplexitätsorientierter Logiken; ihr Kontrollmechanismus ist Einsicht in die Prozesse.

\section{Blinder Fleck 3: die Extension von Kommunikation}

Was umfasst Kommunikation? Und wie wird sie in der Praxis gelebt? Beobachtungen aus der Praxis zeigen (Staller et al. 2019, 2021c), dass Kommunikation in der polizeilichen Lehr- und Einsatzpraxis vergleichsweise begrenzt aufgefasst wird: sowohl in der sachlichen als auch in der kontextuellen Dimension.

Sachlich ist Kommunikation mehr als Reden. „Kommunikatives Handeln und Tun kann sprachliche Zeichen benutzen, muss es aber nicht. Sprache ist nur ein Werkzeug von Kommunikation“ (Reichertz 2020, S. 13). Was kommunikationstheoretisch trivial klingt und in verschiedenen Konzeptionen von Kommunikation zum Ausdruck gebracht wurde ${ }^{1}$, stellt sich in der Praxis der polizeilichen Handlungsempfehlungen anders dar: Auf der einen Seite ist Kommunikation verbale Lösungsoption eines Konfliktes oder Durchsetzungsstrategie einer Maßnahme, auf der anderen Seite ist sie Zwangshandeln mittels Gewalt (Koerner und Staller 2021a). Auch polizeiliche Ausbildungscurricula (z.B. NRW 2018) und praktische Einsatztrainings unterstützen diese Zwei-Welten-Lehre (Staller et al. 2019, 2021c): Kommunikation oder Zwangshandeln - oder wie eine aktuelle Publikation titelt: „Kommunikation statt Gewalt“ (Lorei 2021).

In Bezug auf die kontextuelle Einbettung von Kommunikation zeigt sich der blinde Fleck in der isolierten Betrachtung einer Kommunikation zwischen Bürger*in und Polizei. Kommunikation vollzieht sich im gesellschaftlichen Kontext und ist damit mehr als vor Ort und zeitlich begrenzt. Zur Kommunikationssituation ,gehört immer auch das $D a$ vor und Danach und v. a. das Daneben - gerade weil Situationen oft eingebettet sind in andere Situationen, die pa-

\footnotetext{
1 Z.B. „Man kann nicht nicht kommunizieren“ (Watzlawick et al. 1967) oder die systemtheoretische Konzeptionalisierung als Grundelement jeglicher sozialer Praxis (Luhmann 1984), die in Lehrbüchern zum polizeilichen Bachelorstudiums entsprechend gelehrt wird (Posch \& Werdes, 2016).
} 
rallel verlaufen und mittels Medien miteinander gekoppelt sind" (Reichertz 2020, S. 17). Kommunikation findet in der Tat in einem spezifischen zeitlichen und örtlichen Kontext statt, der sich beispielsweise durch den tagesaktuellen Stand des Vertrauens von Menschen einer spezifischen räumlichen Gegend in die Polizei niederschlägt. Jedoch bedingen das Davor, das Danach und das Daneben, wie die Kommunikation gedeutet und interpretiert wird. Die Interaktion wirkt als Kommunikation wieder zurück in die gesellschaftliche Konstruktion der Realität. Die Analysen zu Clankriminalität (Brauer et al. 2020) und dem Polizieren entlang räumlicher Einteilungen (Hunold et al. 2021) zeigen beispielhaft und eindrucksvoll, wie die Polizei die Möglichkeit besitzt, mittels „Kontrollpraktiken und Gewaltausübung“ (Hunold et al. 2021, S. 22) Realitäten zu konstruieren. Durch diese Art von Kommunikation werden aktiv raum- und gruppenbezogene Images gestaltetet, sodass eigene Erwartungen an Menschen und potenzielle Täter*innen reproduziert werden (Belina und Wehrheim 2011; Hunold et al. 2021). Eine lokal und temporär begrenzte Polizeikontrolle entfaltet Wirkungsmacht in zeitlicher und räumlicher Dimension. Als Kommunikation gestaltet sie gesellschaftliche Wirklichkeiten. Gerade hier zeigt sich auch die Wichtigkeit der Einbettung einzelner Interaktionen in einen langfristigen Handlungsplan, der über eine isolierte Einsatzbewältigung vor Ort hinausgeht. Mit Blick auf eine gerechte und soziale Gesellschaft halten wir es für normativ geboten, polizeiliche Kommunikation im Handeln und Tun an einer gerechten und sozialen Gesellschaft auszurichten (Staller et al. 2021e).

Professionelle Polizeiliche Kommunikation ist die Gänze polizeilichen Interaktions(nicht)verhaltens.

\section{Blinder Fleck 4: Kommunikation von Distanz}

Der kommunikative Konstruktivismus (Keller et al. 2013; Reichertz 2020) weist darauf hin, dass Kommunikation zwischen alter und ego stets unter Anwesenheit eines Dritten der Gesellschaft - stattfindet. Kommunikation hat damit auch immer identitätsbildenden Charakter. Kommunikatives Handeln ist ,sowohl das Medium als auch der Ort, mit dem und an dem Wissen geschaffen wird, und zwar Wissen darüber, wer man selbst, wer der andere und was die Welt für uns ist" (Reichertz 2020, S. 23). In diesem Verständnis ist Kommunikation die Basis gesellschaftlicher Wirklichkeit und dient ,nicht allein der Übermittlung (von Informationen), sondern v. a. der Vermittlung (sozialer Identität und sozialer Ordnung“, Keller et al. 2013, S. 13).

Die Rekonstruktion aus dem, wie sich die gesellschaftliche Wirklichkeit für die Polizei teilweise darstellt (für Analysen: z. B. Behr 2019; Hunold 2019) lässt mehrere Tendenzen erkennen (die sicherlich einer weiteren Analyse bedürfen), welche uns Hinweise auf einen weiteren blinden Fleck in der Kommunikation in spezifischen Situationen (die einer akuten Problemlösung bedürfen) geben: das (Er-)Schaffen von Distanz.

Studien und Analysen weisen darauf hin, dass die Distanz von Polizei und Zivilgesellschaft über die polizeiliche Sozialisation größer wird (Behr 2018; Boivin et al. 2018; Staller et al. 2021a). Die Folgen sind sichtbar.

Auf organisationaler Ebene zeigen sich die folgenden Beispiele:

- Stereotype und Vorurteile, die sich in polizeilichen Praktiken (Abdul-Rahman et al. 2020; Behr 2019) und organisatorischen Entwicklungen (Jimenez et al. 2020) niederschlagen,

- eine ansteigende Militarisierung, welche rigoroser Analysen bedarf, dennoch prima facie sichtbar ist (Kirsch 2017; Naplava 2020),

- Eigendynamik von extrem geschlossenen Systemen wie beispielsweise Spezialeinsatzkommandos (Staller und Koerner 2021c) und

- eine durch das Machtgefälle zwischen Polizei und Bürger*innen sich bekräftigende Sozialdistanz, die im hiesigen Kontext eine Verfremdung der Polizei bedingt; diese Verfremdung schlägt sich in erhöhter Anfälligkeit bestimmter Denkverzerrungen nieder, die eine weitere Verfremdung und Erhöhung der Distanz mit sich bringt und dadurch die polizeiliche Subkultur und das entsprechende, oft bürgerskeptische Werteverständnis perpetuiert (Magee und Smith 2013).

Eine psychosoziale Distanz zeigt sich auch in Interaktionen im Einsatz und die Sichtweise darauf, beispielsweise durch

- das Verharren auf dem eigenen Standpunkt, wenn Änderung der eigenen Position geboten wäre (Klukkert et al. 2008),

- instrumentellen Charakter von perspektivenübernehmenden Gesprächstechniken (,Dann fügt es sich besser unseren Maßnahmen“) (Bernt und Kuhleber 1991),

- das Verhalten im Umgang mit Menschen verschiedener Gruppen: ,people of color“" (Abdul-Rahman et al. 2020), Menschen ohne Obdach (Kyprianides et al. 2020) oder Menschen mit psychischen Erkrankungen (Finzen 2014).

Distanz ist in der Kommunikation der Polizei v. a. über das Konzept der „Eigensicherung“ mit Sicherheit konnotiert (Koerner und Staller 2021b). Während in spezifischen Situationen körperliche Distanz zielführend ist (aber nicht ausschließlich), stellt sich auf psychologischer und gesellschaftlicher Ebene das Primat von Distanz weitaus problematischer da. Der Kontrapart, Nähe und Kontakt,

- ermöglicht den Abbau von Vorurteilen und Stereotypen (Pettigrew 2016), 
- ermöglicht als implizite Basis von Kommunikationsmodellen und -strategien eine empathische, perspektivenannehmende, vorurteilsfreie Verbalkommunikation in Einsatz- und Krisensituationen (Vecchi et al. 2019; Zaiser et al. 2021; Zaiser und Staller 2015) und

- trägt zum Aufbau von Vertrauen und Zusammenhalt zwischen Polizei und Zivilgesellschaft bei, was wiederum nahhaltige Effekte auf die Polizeiarbeit positiv beeinflusst (Giles et al. 1991).

Mit Fokus auf den blinden Fleck der Distanzkommunikation gilt es, durch polizeiliche Kommunikation soziale Distanzen zu verringern. Dies umfasst beispielsweise das Verwenden einer inklusiven Sprache, den Einsatz gegen diskriminierende polizeiliche Praktiken (z.B. ,racial und social profiling“), das Einlassen und das aktive Aufsuchen der Erlebniswelt und Perspektiven anderer Menschen, Verwenden von nichturteilender Kommunikation und vieles mehr. Die Distanzverringerung ist ein wesentlicher Bestandteil einer Polizeiarbeit, welche am Modell der Bürgerpolizei (Naplava 2020) ausgerichtet ist.

Professionelle polizeiliche Kommunikation verringert soziale Distanzen.

\section{Blinder Fleck 5: Kommunikationsasymmetrie in Bezug auf die Erwartungshaltung}

Jenseits der genannten Asymmetrie polizeilicher Kommunikation (Option zur legitimierter Gewaltausübung und Änderungswunsch) existiert eine weitere Asymmetrie zwischen alter und ego, die für eine professionelle Kommunikation notwendig ist.

Mit dem staatlichen Gewaltmonopol geht die Verantwortung einher, die sich in unterschiedlichen Erwartungen und auch in unterschiedlichen Kapazitäten widerspiegelt. Polizist*innen sollten, im Sinne der langfristigen strategischen Ausrichtung, über gewalt- und konfliktreduzierendes Wissen und/oder Können verfügen. Dies schließt auch die Fähigkeit zu Optionen unmittelbaren Zwangs mit ein (Staller et al. 2021e). Die Asymmetrie zeigt sich darin, dass die andere Partei (ego) diese Kapazität möglicherweise, auch aufgrund akuter situativer Umstände (z.B. Alkoholintoxikation, psychische Störung, Emotionalität etc.), nicht hat. Eine solche Kapazität kann von ihr aber auch nicht erwartet werden. Das Tolerieren von wahrgenommener Respektlosigkeit, das Infragestellen von Autorität sowie verbale und körperliche Gewalt werden damit explizit eine Kommunikationsoption.

Die Debatten um das Antwortverhalten auf eine (gefühlte) Erodierung des Respekts und der Autorität (Naplava 2020) weisen darauf hin, dass diese Asymmetrie in der kommunikativen Ausgangssituation so nicht immer wahrgenommen wird. Autoritäts- und Respektverlusten wird mit
Härte und „Robustheit“ (Behr 2018) begegnet, was ihren Ausdruck in einer ansteigend diagnostizierten Militarisierung findet (Naplava 2020). Das situative Tolerieren, basierend auf einem kontextuellen und reflexiven Hinterfragen der eigenen Wahrnehmung und dessen Einordnung, widerspricht dieser Stoßrichtung.

Professionelle Polizeiliche Kommunikation ist sich der Erwartungsasymmetrie in Konfliktsituationen bewusst. Aus großer Macht (im Sinne des Gewaltmonopols) folgt große Verantwortung.

\section{Blinder Fleck 6: Unterschätzen des eigenen blinden Flecks}

Das Erkennen von blinden Flecken, also vom Ausschlussbereich eigener Beobachtungen, ist nur durch eine Änderung der eigenen Beobachterperspektive, insbesondere durch eine zeitlich (man selbst) oder räumlich (durch andere) versetze Reflexion möglich (Körner 2015). Durch Beobachtung der eigenen Prozesse und derjenigen Prozesse, die zu diesen Bewertungen führen (Staller et al. 2021d), besteht die Möglichkeit, sich darbietende Sachverhalte und Konflikte in einem anderen Licht zu sehen. Dies bietet auch die Möglichkeit, den gemeinsamen Nenner aus bis dato als unvereinbar geltenden Positionen zu sehen; ein Kernprozess moderner Verhandlungsführung (Fisher et al. 2011).

Die Annahmen des Gewinns von Reflexivität führt nicht automatisch zu dessen Praxis. Als anstrengende kognitive Operation ist sie ebenfalls einer kognitiven Verzerrung unterworfen: Uns fällt es leichter, blinde Flecken bei anderen zu identifizieren als unsere eigenen (Pronin et al. 2002). In Kombination mit organisationalen Sozialisationseffekten und dort tradierten Fehlschlüssen (und weiteren kognitiven Verzerrungen) entsteht daraus eine immanente Systemlogik, die schwer zu durchbrechen ist (Staller et al. 2021). Sich haltende blinde Flecken sind damit eine wesentliche Eigenschaft des Systems Polizei. Wir identifizieren Potenzial für Veränderung in einer (zu erwerbenden) systemimmanenten Reflexivität und externen Beobachtungsperspektiven (Negnal et al. 2019) sowie strukturellen Prozessen, die beides miteinander koppelt.

Für die polizeiliche Kommunikation heißt das:

- Individuelle und organisatorische (Nicht-)Handlungen sind Kommunikation und haben als solche Bedeutungswert (welcher jeweils von alter und ego selektiv wahrgenommen und verarbeitet wird); sie sollen reflexiv in den Blick genommen werden (Staller et al. 2021a; Staller und Koerner 2021a).

- Eigene kommunikative Handlungen sind selbst das Ergebnis individueller und organisationaler Sozialisationsprozesse. Die Entwicklung und Wirkung der dadurch ent- 
standenen handlungsleitenden Annahmen sind kritisch in den Blick zu nehmen (Staller et al. 2021d).

- Kommunikation ist Veränderung und erfordert die Bereitschaft von ego sich auf alter einzustellen, was in der Ausprägung und vor dem Hintergrund eigener relevanter Selektionskriterien passiert (Luhmann 1984): Was wird wie warum mitgeteilt und verstanden? Welche anderen Optionen werden dabei ausgeschlossen? Ein prominentes Beispiel hierfür ist der fundamentale Attributionsfehler (Gawronski 2004), bei dem Kommunikation und Handeln des Gegenübers ohne Rücksicht auf situationsund sozialisierungsbedingte Faktoren interpretiert werden. Die situative Perspektive wird durch Wahrnehmung einer vermeintlichen Ungerechtigkeit konsolidiert und schreibt dem Gegenüber oft böswilliges oder egozentrisches Verhalten zu. Im polizeilichen Kontext wird der fundamentale Attributionsfehler sowohl auf der Erfahrungsseite der Bürger*innen als auch der Polizist*innen wahrgenommen.

Sich verstehen heißt, vor dem Hintergrund individueller und organisationaler blinder Flecken in erster Linie sich selbst zu verstehen. Reflexivität als notwendige Voraussetzung einer auf Einsicht basierenden professionellen polizeilichen Kommunikation.

Professionelle Polizeiliche Kommunikation nimmt sich reflexiv selbst in den Blick.

\section{Fazit}

Polizeiliche Kommunikation hat Reflexionsbedarf. Basierend auf einer pragmatistischen Forschungs- und Analyseperspektive identifizierten wir 6 blinde Flecken polizeilicher kommunikativer Praxis, die wir als Leitgedanken in Bildungsveranstaltungen polizeilicher Kommunikation überantworten:

1. Professionelle Polizeiliche Kommunikation ist sich den sie beeinflussenden kognitiven Verzerrungen und Fehlurteilen bewusst.

2. Professionelle Polizeiliche Kommunikation bedient sich komplexitätsorientierter Logiken; ihr Kontrollmechanismus ist Einsicht in die Prozesse.

3. Professionelle Polizeiliche Kommunikation ist die Gänze polizeilichen Interaktions(nicht)verhaltens.

4. Professionelle polizeiliche Kommunikation verringert soziale Distanzen.

5. Professionelle Polizeiliche Kommunikation ist sich der Erwartungsasymmetrie in Konfliktsituationen bewusst.

6. Professionelle Polizeiliche Kommunikation nimmt sich reflexiv selbst in den Blick.
Als Qualifizierungen einer Professionellen Polizeilichen Kommunikation hoffen wir damit zu einer Professionalisierung polizeilicher Praxis beizutragen.

Funding Open Access funding enabled and organized by Projekt DEAL.

Interessenkonflikt M. S. Staller, S. Koerner und B. Zaiser geben an, dass kein Interessenkonflikt besteht.

Open Access Dieser Artikel wird unter der Creative Commons Namensnennung 4.0 International Lizenz veröffentlicht, welche die Nutzung, Vervielfältigung, Bearbeitung, Verbreitung und Wiedergabe in jeglichem Medium und Format erlaubt, sofern Sie den/die ursprünglichen Autor(en) und die Quelle ordnungsgemäß nennen, einen Link zur Creative Commons Lizenz beifügen und angeben, ob Änderungen vorgenommen wurden.

Die in diesem Artikel enthaltenen Bilder und sonstiges Drittmaterial unterliegen ebenfalls der genannten Creative Commons Lizenz, sofern sich aus der Abbildungslegende nichts anderes ergibt. Sofern das betreffende Material nicht unter der genannten Creative Commons Lizenz steht und die betreffende Handlung nicht nach gesetzlichen Vorschriften erlaubt ist, ist für die oben aufgeführten Weiterverwendungen des Materials die Einwilligung des jeweiligen Rechteinhabers einzuholen.

Weitere Details zur Lizenz entnehmen Sie bitte der Lizenzinformation auf http://creativecommons.org/licenses/by/4.0/deed.de.

\section{Literatur}

Abdul-Rahman L, Grau HE, Klaus L, Singelnstein T (2020) Rassismus und Diskriminierungserfahrungen im Kontext polizeilicher Gewaltausübung. Zweiter Zwischenbericht zum Forschungsprojekt „Körperverletzung im Amt durch Polizeibeamt*innen“ (KviAPol). Ruhr-Universität Bochum, Bochum

Behr R (2018) „Die Polizei muss ... an Robustheit deutlich zulegen“: Zur Renaisssance aggressiver Maskulinität in der Polizei. In: Loik D (Hrsg) Kritik der Polizei. Campus, Frankfurt/M., S $165-180$

Behr R (2019) Verdacht und Vorurteil. Die polizeiliche Konstruktion der ,gefährlichen Fremden“. In: Howe C, Ostermeier L (Hrsg) Polizei und Gesellschaft - Transdisziplinäre Perspektiven zu Methoden, Theorie und Empirie reflexiver Polizeiforschung, Bd. 3. Springer VS, Wiesbaden, S 17-45 https://doi.org/10.1007/978-3658-22382-3_2

Belina B, Wehrheim J (2011) „Gefahrengebiete“ - Durch die Abstraktion vom Sozialen zur Reproduktion gesellschaftlicher Strukturen. SozProb 22(2):207-230

Bennell C, Alpert G, Andersen JP, Arpaia J, Huhta J, Kahn KB, Khanizadeh A, McCarthy M, McLean K, Mitchell RJ, Nieuwenhuys A, Palmer A, White MD (2021) Advancing police use of force research and practice: urgent issues and prospects. Legal Criminol Psychol. https://doi.org/10.1111/lcrp.12191

Berkes F, Berkes MK (2009) Ecological complexity, fuzzy logic, and holism in indigenous knowledge. Futures 41(1):6-12. https://doi. org/10.1016/j.futures.2008.07.003

Berne E (1961) Transactional analysis in psychotherapy. Grove Press, New York

Bernt P, Kuhleber HW (1991) Das deeskalative Einsatzmodell. Polizei 9:219-235

Boivin R, Faubert C, Gendron A, Poulin B (2018) The 'us vs them' mentality: a comparison of police cadets at different stages of their training. Police Pract Res 5(1):1-13. https://doi.org/10.1080/ 15614263.2018.1555480

Boxer P, Brunson RK, Gaylord-Harden N, Kahn K, Patton DU, Richardson J, Rivera LM, Lee JRS, Staller MS, Krahé B, Dubow EF, 
Parrott D, Algrim K (2021) Addressing the inappropriate use of force by police in the United States and beyond: a behavioral and social science perspective. Aggr Behav 47(5):505-512. https:// doi.org/10.1002/ab.21970

Brauer E, Dangelmeier T, Hunold D (2020) Die diskursive Konstruktion von Clankriminalität. Schriften zur Empirischen Polizeiforschung, Bd. 26

Fachhochschule für öffentliche Verwaltung NRW (2018) Modulhandbuch Bachelorstudiengang PVD 2016 ab dem Einstellungsjahr 2018

Feil N (1989) Validation: an empathic approach to the care of dementia. Clin Gerontol 8(3):89-94

Finzen A (2014) Polizei-Interventionen? Jeder dritte Tote war psychisch krank. Psychiat Prax 41(01):50-52. https://doi.org/10. 1055/s-0033-1336908

Fisher R, Ury W, Patton B (2011) Getting to yes. Penguin Books, New York

Frenkel MO, Giessing L, Egger-Lampl S, Hutter V, Oudejans R, Kleygrewe L, Jaspaert E, Plessner H (2020) The impact of the COVID19 pandemic on European police officers: stress, demands and coping resources. J Crim Justice. https://doi.org/10.1016/j.jcrimjus. 2020.101756

Frenkel MO, Giessing L, Jaspaert E, Staller MS (2021) Mapping demands: How to prepare police officers to cope with pandemicspecific stressors. Eur Law Enforc Res Bull 21:11-22

Gawronski B (2004) Theory-based bias correction in dispositional inference: the fundamental attribution error is dead, long live the correspondence bias. Eur Rev Soc Psychol 15(1):183-217. https://doi.org/10.1080/10463280440000026

Giles H, Coupland N, Coupland J (1991) Accommodation theory: communication, context, and consequence. In: Giles H, Coupland J, Coupland N (Hrsg) Contexts of accommodation. Cambridge University Press, Cambridge, S 1-68 https://doi.org/10. 1017/cbo9780511663673.001

Hermanutz M, Spöcker W (2012) Kommunikation mit den Bürgern bei polizeilichen Routinetätigkeiten. In: Moderne Polizeipsychologie in Schlüsselbegriffen, 3. Aufl. Richard Boorberg, Stuttgart, S $147-155$

Hunold D (2019) „Wer hat jetzt die größeren Eier?!“ - Polizeialltag, hegemoniale Männlichkeit und reflexive Ethnografie. In: Howe C, Ostermeier L (Hrsg) Polizei und Gesellschaft - Transdisziplinäre Perspektiven zu Methoden, Theorie und Empirie reflexiver Polizeiforschung. Springer VS, Wiesbaden, S 47-69 https://doi.org/ 10.1007/978-3-658-22382-3_3

Hunold D, Dangelmaier T, Brauer E (2021) Soziale Ordnung und Raum - Aspekte polizeilicher Raumkonstruktion. SozProb 32(1):19-44. https://doi.org/10.1007/s41059-020-00070-1

Innenministerkonferenz (2011) Leitfaden 371: Eigensicherung

Jimenez T, Helm PJ, Wilinson A, Arndt J (2020) Racial prejudice predicts police militarization https://doi.org/10.31234/osf.io/7uhge

Kade J (2004) Erziehung als pädagogische Kommunikation. In: Lenzen D (Hrsg) Irritationen des Erziehungssystems: Pädagogische Resonanzen auf Niklas Luhmann. Surhkamp, Frankfurt/M., S $199-232$

Keller R, Knoblauch H, Reichertz J (2013) Der Kommunikative Konstruktivismus als Weiterführung des Sozialkonstruktivismus - eine Einführung in den Band. In: Keller R, Reichertz J, Knobloch H (Hrsg) Kommunikativer Konstruktivismus, Theoretische und empirische Arbeiten zu einem neuen wissenssoziologischen Ansatz. Springer VS, Wiesbaden, S 9-21 https://doi.org/10.1007/978-3531-19797-5 1

Kern P (2017) Polizei und taktische Kommunikation. Springer, Wiesbaden https://doi.org/10.1007/978-3-658-17197-1

Kirsch M (2017) Militarisierung der Polizei: Massive Aufrüstung im Namen der Terrorabwehr. Informationsstelle Militarisierung, Tübingen

Klukkert A, Ohlemacher T, Feltes T (2008) Torn between two targets: German police officers talk about the use of force. Crime Law
Soc Change 52(2):181-206. https://doi.org/10.1007/s10611-0089178-5

Koerner S, Staller MS (2021a) Die Tools der Straße II: Eigensicherung. Dtsch Polizeibl 39(4):13-15

Koerner S, Staller MS (2021b) Eigensicherung, reflektiert. In: Staller MS, Koerner S (Hrsg) Handbuch polizeiliches Einsatztraining: Professionelles Konfliktmanagement (in press)

Körner S (2009) Reflexion von Kontingenz und Kontingenz der Reflexion: Ein systemtheoretischer Kommentar. In: Lüsebrink I, Krieger C, Wolters P (Hrsg) Schulinterne Evaluation. Impulse zur Selbstvergewisserung aus sportpädagogischer Perspektive. Sportverlag Strauß, Hellenthal, S 141-160

Körner S (2015) Reflexive Mechanismen und Sportwissenschaft. In: Schürmann V (Hrsg) Reflexive Sportwissenschaft. Lehmanns Media, Berlin, S 129-143

Körner S (2017) Alles Inklusion, oder was? Sport Ges 14(1):5-27. https://doi.org/10.1515/sug-2017-0002

Körner S, Staller MS (2018) Pedagogy of terrorism. Mujahid Guide revisited. J Polic Intell Count Terror 13(2):332-344. https://doi. org/10.1080/18335330.2018.1503700

Krauss RM, Fussell SR (1996) Social psychological models of interpersonal communication. In: Higgins ET, Kruglanski A (Hrsg) Social psychology: handbook of basic principles. Guilford, New York

Kyprianides A, Stott C, Bradford B (2020) 'Playing the game': power, authority and procedural justice in interactions between police and homeless people in London. Br J Criminol. https://doi.org/ $10.1093 / \mathrm{bjc} / \mathrm{azaa} 086$

Lorei C (2021) Kommunikation statt Gewalt. Kriminalistik 75(1):16-23

Luhmann N (1981) Die Unwahrscheinlichkeit der Kommunikation. In: Soziales System, Gesellschaft, Organisation. Soziologische Aufklärung, Bd. 3, S 25-34 https://doi.org/10.1007/978-3-66301340-2_2

Luhmann N (1984) Soziale Systeme. Suhrkamp, Frankfurt/M.

Luhmann N (1990) Die Wissenschaft der Gesellschaft. Suhrkamp, Frankfurt/M

Luhmann N (1995) Was ist Kommunikation? In: Luhmann N (Hrsg) Die Soziologie und der Mensch. Soziologische Aufklärung, Bd. 6. Westdeutscher Verlag, Opladen, S 113-124

Luhmann N, Schorr KE (1982) Das Technologiedefizit der Erziehung und der Pädagogik. In: Luhmann N, Schorr KE (Hrsg) Zwischen Technologie und Selbstreferenz. Suhrkamp, Frankfurt/M., S $11-41$

Magee JC, Smith PK (2013) The social distance theory of power. Pers Soc Psychol Rev 17(2):158-186. https://doi.org/10.1177/ 1088868312472732

Naplava T (2020) „Militarisierung“ als Antwort auf „mangelnden Respekt"? Ein soziologischer Beitrag zur Diskussion um einen Paradigmenwechsel der Polizei in Deutschland. In: Hunold D, Ruch A (Hrsg) Polizeiarbeit zwischen Praxishandeln und Rechtsordnung. Springer, Wiesbaden, S 165-183 https://doi.org/10.1007/978-3658-30727-1_8

Nassehi A, Saake N (2002) Kontingenz: Methodisch verhindert oder beobachtet? Z Soziol 31(1):66-86

Negnal D, Howe C, Porsché Y (2019) Polizei und Kritik. Ein Beitrag der Ethnografie. In: Howe C, Ostermeier L (Hrsg) Polizei und Gesellschaft. Springer VS, Wiesbaden https://doi.org/10.1007/9783-658-22382-3_8

Pettigrew TF (2016) In pursuit of three theories: authoritarianism, relative deprivation, and intergroup contact. Annu Rev Psychol 67(1):1-21. https://doi.org/10.1146/annurev-psych-122414033327

Posch T, Werdes B (Hrsg) (2016) Polizeipsychologie: Ein Lehrbuch für das Bachelorstudium Polizei. Hogrefe

Pronin E, Lin DY, Ross L (2002) The bias blind spot: perceptions of bias in self versus others. Pers Soc Psychol Bull 28(3):369-381. https://doi.org/10.1177/0146167202286008 
Reichertz J (2010) Kommunikationsmacht. Was ist Kommunikation und was vermag sie? Und weshalb vermag sie das? https://doi. org/10.1007/978-3-531-91915-7

Reichertz J (2020) Einleitung: Grenzen der Kommunikation - Kommunikation an den Grenzen. In: Reichertz J (Hrsg) Grenzen der Kommunikation - Kommunikation an den Grenzen. Velbrück Wissenschaft, Weilerswist, S 11-27

Rogers CR, Farson RE (1957) Active listening. Industrial Relations Center of the University of Chicago, Chicago

Rorty R (2003) Wahrheit und Fortschritt. Suhrkamp, Frankfurt/M.

Rosenberg MB (2003) Nonviolent communication: a language of life, 2. Aufl. Puddle Dancer Press, Encinitas

Schön DA (1983) The reflective practitioner: how professionals think in action. Basic Books, New York

Schuck AM (2019) Political authority, social control and public policy. Public Policy Gov 31:63-77. https://doi.org/10.1108/s2053769720190000031007

Schulz von Thun F (1981) Miteinander Reden. Rowolth, Hamburg

Seidensticker K (2021) Aggressive Polizeimännlichkeit: Noch hegemonial, aber neu begründet. Bürgerrechte \& Polizei / CILIP, Bd. 126

Staller MS, Koerner S (2021a) Evidence-based policing or reflexive policing: a commentary on Koziarski and Huey. Int J Comp Appl Crim Justice. https://doi.org/10.1080/01924036.2021.1949619

Staller MS, Koerner S (2021b) Kontingenz als Stimulanz: Vom Nutzen epistemologischer Verschlingungen von Forschung und Gegenstand am Beispiel des Constraints-Led-Approach. In: Zander B, Rode D, Schiller D, Wolf D (Hrsg) Qualitatives Forschen in der Sportpädagogik - Beiträge zu einer reflexiven Methodologie

Staller MS, Koerner S (2021c) "We have to end this now": A case example of immunizing strategies of a Special Operation Force against threatening knowledge. https://doi.org/10.13140/rg.2.2. 18943.53922

Staller MS, Koerner S (2021d) Science and police use of force training: a critical commentary. Perform Improv Q : (accepted)

Staller MS, Körner S, Heil V, Kecke A (2019) Mehr gelernt als geplant? Versteckte Lehrpläne im Einsatztraining. In: Frevel B, Schmidt P (Hrsg) Empirische Polizeiforschung XXII: Demokratie und Menschenrechte - Herausforderungen für und an die polizeiliche Bildungsarbeit. Verlag für Polizeiwissenschaft, Frankfurt/M., S 132-149

Staller MS, Koerner S, Heil V (2021a) Guardian oder Warrior? Überlegungen zu polizeilichen Grundeinstellungen [Guardian or Warrior? Reflections on basic police attitudes. In: Staller MS, Koerner S
(Hrsg) Handbuch polizeiliches Einsatztraining. Springer, Berlin Heidelberg (in press)

Staller MS, Koerner S, Heil V, Abraham A, Poolton J (2021b) Police recruits' perception of skill transfer from training to the field. Int J Police Sci Manag. (accepted)

Staller MS, Koerner S, Heil V, Klemmer I, Abraham A, Poolton J (2021c) The structure and delivery of police use of force training-A German case study. European Journal for Security Research. https://doi.org/10.1007/s41125-021-00073-5

Staller MS, Koerner S, Zaiser B (2021d) Der/die reflektierte Praktiker*in: Reflektieren als Polizist*in und Einsatztrainer*in. In: Staller MS, Koerner S (Hrsg) Handbuch polizeiliches Einsatztraining: Professionelles Konfliktmanagement. Springer, Wiesbaden

Staller MS, Koerner S, Zaiser B (2021e) Mehr GeredE - Ein Plädoyer für die Ablösung des Deeskalierenden Einsatzmodells [More GeredE-A plea for the replacement of the Deescalating Deployment Model]. Polizei 111(2):72-81

Staller MS, Zaiser B, Koerner S (2021f) The Problem of Entanglement: Biases and Fallacies in Police Conflict Management. Int J Police Sci Manag. (accepted)

Vecchi GM, Hasselt VV, Romano SJ (2005) Crisis (hostage) negotiation: current strategies and issues in high-risk conflict resolution. Aggress Violent Behav 10(5):533-551. https://doi.org/10.1016/j. avb.2004.10.001

Vecchi GM, Wong GKH, Wong PWC, Markey MA (2019) Negotiating in the skies of Hong Kong: the efficacy of the behavioral influence stairway model (BISM) in suicidal crisis situations. Aggress Violent Behav 48:230-239. https://doi.org/10.1016/j.avb.2019. 08.002

Watzlawick P, Beavin JH, Jackson DD (1967) Pragmatics of human communication: a study of interactional patterns, pathologies, and paradoxes. W. W. Norton, New York

Werner S (2019) Professionalität. https://www.socialnet.de/lexikon/ Professionalitaet (Socialnet Lexikon). Zugegriffen: 25. Juli 21

Zaiser B, Staller MS (2015) The word is sometimes mightier than the sword: rethinking communication skills to enhance officer safety. J Law Enforc 4(5):1-17

Zaiser B, Staller MS, Koerner S (2021) Die Tools der Straße I: Verbale Kommunikation im Einsatz. Dtsch Polizeibl 39(4):9-12 